\title{
Horizontal Transmission of Chronic Wasting Disease in Reindeer
}

\author{
S. Jo Moore, Robert Kunkle, \\ M. Heather West Greenlee, Eric Nicholson, \\ Jürgen Richt, Amir Hamir, ${ }^{1}$ \\ W. Ray Waters, Justin Greenlee
}

We challenged reindeer by the intracranial route with the agent of chronic wasting disease sourced from white-tailed deer, mule deer, or elk and tested for horizontal transmission to naive reindeer. Reindeer were susceptible to chronic wasting disease regardless of source species. Horizontal transmission occurred through direct contact or indirectly through the environment.

$\mathrm{R}$ eindeer are susceptible to chronic wasting disease (CWD) after experimental oral challenge (1), and recently, CWD was identified in a free-ranging reindeer in Norway $(2,3)$. Horizontal transmission is the primary mode of CWD transmission in deer. Direct horizontal transmission occurs when naive animals are exposed to infectious excreta (i.e., saliva, urine, feces) during close contact with CWD-affected animals (reviewed in 4). Indirect horizontal transmission occurs through exposure to environments contaminated with infectious material (e.g., excreta or decomposed carcasses) $(5,6)$.

The Eurasian reindeer (Rangifer tarandus tarandus) is closely related to the North American caribou (R. t. caribou, $R$. t. granti, $R$. t. groenlandicus). In North America, overlapping geographic ranges of free-ranging populations of potentially CWD-infected white-tailed deer (Odocoileus virginianus), mule deer (O. hemionus), or elk (Cervus elaphus nelsoni) present a risk for horizontal transmission to caribou. Exposure also could occur in farmed populations where contact occurs between reindeer and captive and/or free-ranging CWD-affected cervids. We investigated the transmission of CWD from white-tailed deer, mule deer, or elk to reindeer through the intracranial route and assessed them for direct and indirect horizontal transmission to uninoculated sentinels.

\section{The Study}

In 2005, we challenged reindeer fawns from a farm in Alaska, USA, where CWD had never been reported, by intracranial

Author affiliations: US Department of Agriculture, Ames, lowa, USA (S.J. Moore, R. Kunkle, E. Nicholson, J. Richt, A. Hamir, W.R. Waters, J. Greenlee): Iowa State University, Ames (M.H. West Greenlee) inoculation (7) with pooled brain material from CWDaffected elk from South Dakota $\left(\mathrm{CWD}^{\mathrm{elk}}\right)$, CWD-affected mule deer from Wyoming $\left(\mathrm{CWD}^{\mathrm{md}}\right)$, or CWD from whitetailed deer from Wisconsin combined with brain material from experimentally challenged white-tailed deer (CWD $\left.{ }^{\text {wtd }}\right)$ (Table 1; online Technical Appendix, http://wwwnc.cdc. gov/EID/article/22/12/16-0635-Techapp1.pdf). Additional uninoculated fawns served as negative controls, controls for indirect transmission, and controls for direct transmission (Table 1; online Technical Appendix). We determined the prion protein gene (PRNP) genotype of each fawn (online Technical Appendix), and we tried to ensure that each PRNP genotype was present in each group (Table 2, http://wwwnc. cdc.gov/EID/article/22/12/16-0635-T1.htm). Control reindeer were housed in the same barn as inoculated reindeer but in separate pens that prevented direct physical contact (i.e., nose-to-nose) between control and inoculated animals (online Technical Appendix Figure 1). Indirect and direct contact control groups were formed 25 months after intracranially challenged reindeer were inoculated (online Technical Appendix Figure 1, panel B).

Clinical signs consistent with CWD were first observed 20.9 months after inoculation (Table 2). Common clinical features included found dead without clinical signs noted, loss of body condition, recumbency, and lethargy (Table 2; online Technical Appendix).

At death, a full necropsy was performed on all reindeer. Two sets of tissue samples were collected: 1 set was fixed in $10 \%$ buffered formalin, embedded in paraffin wax, sectioned at $5 \mu \mathrm{m}$ for microscopy examination after hematoxylin and eosin staining or immunohistochemical staining using primary antibody F99/96.7.1 (online Technical Appendix). A second set of tissues was frozen, and selected tissues were used for immunodetection of scrapie prion protein $\left(\mathrm{PrP}^{\mathrm{Sc}}\right)$ by Western blot (brain tissue only) as described previously (7) but with some modifications, or an ELISA (brainstem and/or retropharyngeal lymph node) using a commercial kit (IDEXX HerdChek BSE-Scrapie Antigen ELISA; IDEXX, Westbrook, ME, USA) according to the manufacturers' instructions (online Technical Appendix).

In the intracranially inoculated groups, when intercurrent deaths were excluded, reindeer with the NN138 polymorphism (reindeer nos. 2, 6, and 12) had the shortest survival times in each group (Table 2). Different inocula did not produce significantly different survival times (log-rank 
Table 1. Animal data for reindeer (Rangifer tarandus tarandus) in a study of transmission of CWD*

\begin{tabular}{|c|c|c|c|c|c|c|c|}
\hline \multirow{2}{*}{$\begin{array}{l}\text { Group } \\
\text { no./animal no. }\end{array}$} & \multicolumn{5}{|c|}{ Genotype codon } & \multirow[b]{2}{*}{ Infectivity source } & \multirow[b]{2}{*}{ Exposure route } \\
\hline & 002 & 129 & 138 & 169 & 176 & & \\
\hline \multicolumn{8}{|l|}{1} \\
\hline 1 & MV & SG & NS & MV & NN & CWD wtd & Intracranial \\
\hline 2 & VV & GG & NN & VV & NN & $\mathrm{CWD}^{\mathrm{wtd}}$ & Intracranial \\
\hline 3 & VV & GG & NS & VV & ND & $\mathrm{CWD}^{\text {wtd }}$ & Intracranial \\
\hline 4 & VV & GG & NS & VV & NN & $\mathrm{CWD}^{\mathrm{wtd}}$ & Intracranial \\
\hline 5 & MV & SG & SS & MV & ND & CWD wtd & Intracranial \\
\hline \multicolumn{8}{|l|}{2} \\
\hline 6 & VV & GG & NN & VV & NN & CWD elk & Intracranial \\
\hline 7 & MV & SG & NS & MV & NN & CWD $^{\text {elk }}$ & Intracranial \\
\hline 8 & VV & GG & NS & VV & NN & CWDelk & Intracranial \\
\hline 9 & VV & GG & NS & VV & ND & CWDelk & Intracranial \\
\hline 10 & NA & SG & SS & MV & NN & CWD elk & Intracranial \\
\hline \multicolumn{8}{|r|}{ 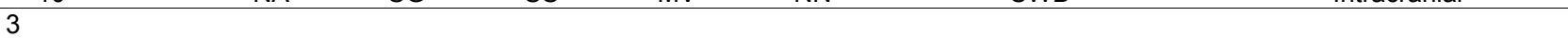 } \\
\hline 11 & MV & SG & NS & MV & NN & $\mathrm{CWD}^{\mathrm{md}}$ & Intracranial \\
\hline 12 & VV & GG & NN & VV & NN & $\mathrm{CWD}^{\mathrm{md}}$ & Intracranial \\
\hline 13 & VV & GG & SS & VV & DD & $\mathrm{CWD}^{\mathrm{md}}$ & Intracranial \\
\hline 14 & MV & SG & SS & MV & NN & $\mathrm{CWD}^{\mathrm{md}}$ & Intracranial \\
\hline 15 & VV & GG & NS & VV & ND & $\mathrm{CWD}^{\mathrm{md}}$ & Intracranial \\
\hline \multicolumn{8}{|l|}{4 direct } \\
\hline 16 & VV & GG & NN & VV & NN & Horizontal (CWD wtd $)$ & Cohoused with group 1 \\
\hline 17 & VV & GG & NN & VV & NN & Horizontal (CWD wid $)$ & Cohoused with group 1 \\
\hline 18 & VV & GG & NN & VV & NN & Horizontal (CWD wid $)$ & Cohoused with group 1 \\
\hline 19 & NA & SG & NS & MV & NN & Horizontal (CWD ${ }^{\text {wtd }}$ ) & Cohoused with group 1 \\
\hline \multicolumn{8}{|l|}{4 indirect } \\
\hline 20 & MM & SS & SS & MM & NN & Horizontal (CWD $\left.{ }^{m d}\right)$ & Housed adjacent to group 3 \\
\hline 21 & VV & GG & NN & VV & NN & Horizontal (CWD ${ }^{m d}$ ) & Housed adjacent to group 3 \\
\hline \multicolumn{8}{|l|}{4 neg. controls } \\
\hline 22 & VV & GG & NS & VV & NN & NA & NA \\
\hline 23 & MV & SG & SS & MV & NN & NA & NA \\
\hline
\end{tabular}

test, $p=0.0931$ ), but we observed differences in the amount of vacuolation and $\mathrm{PrP}^{\mathrm{Sc}}$ in the brain at the clinical stages of disease in $\mathrm{CWD}^{\mathrm{wtd}}$ - and $\mathrm{CWD}^{\mathrm{elk}}$-inoculated reindeer, compared with $\mathrm{CWD}^{\mathrm{md}}$-inoculated reindeer (Table 2; online Technical Appendix). In the indirect contact animals, $\operatorname{PrP}^{\mathrm{Sc}}$ was present in the brain but restricted to the dorsal motor nucleus of the vagus nerve and area postrema.

We observed different patterns of $\mathrm{PrP}^{\mathrm{Sc}}$ deposition in the brain (Figure 1, panels A-D; online Technical Appendix), the most striking of which was dominated by aggregated deposits of various sizes, including plaque-like deposits (Figure 1, panels A,B). This pattern was seen in reindeer with the NS138 NN176 (no. 8, CWD ${ }^{\text {elk; }}$ no. 13, $\mathrm{CWD}^{\mathrm{md}}$ ) or SS138 DD176 (no. 4, CWD ${ }^{\text {wtd }}$ ) genotypes. With regard to immunoreactivity in the retina (Figure 1, panels E, F; online Technical Appendix), in 2 of 3 reindeer with aggregated deposits in the brain (nos. 8 and 13), aggregated immunoreactivity also was observed in the inner plexiform layer of the retina (Figure 1, panel f).

Reindeer that were negative by immunohistochemical analysis in brain also were negative by Western blot and ELISA. Different Western blot migration patterns were observed in $\mathrm{PrP}^{\mathrm{Sc}}$-positive animals (Figure 2), but we found no clear association between migration pattern and challenge group or $P R N P$ genotype.
$\mathrm{PrP}^{\mathrm{Sc}}$ was widespread in lymphoid tissues from most reindeer (Table 2; online Technical Appendix). Reindeer with the NS138 genotype had a significantly lower average percentage of lymphoid follicles positive than did reindeer with NN138 (analysis of variance, $\mathrm{p}=0.003$ ) or SS138 (p $=0.003$ ) deer. Excluding intercurrent deaths, $\mathrm{PrP}^{\mathrm{Sc}}$ was detected in all $4 \mathrm{CWD}^{\text {wtd }}$-challenged reindeer, all $5 \mathrm{CWD}^{\text {elk }}$ challenged reindeer, all $4 \mathrm{CWD}^{\mathrm{md}}$-challenged reindeer, both indirect contact reindeer, and 2 of 4 direct contact reindeer (Table 2).

\section{Conclusions}

Potential sources of infectivity for direct contact animals include urine, feces, and saliva from their $\mathrm{CWD}^{\text {wtd }}$-challenged pen-mates, as has been shown for CWD-affected white-tailed deer $(6,8,9)$. Pinpointing the source of infectivity in the indirect contact group is more difficult. Infectious prions can travel at least $30 \mathrm{~m}$ in airborne particulate (10), but because the negative control reindeer in the pen adjacent to the indirect contact reindeer did not become positive, a more direct route of transmission is likely in this case. Penning, feeding, and watering protocols were designed to prevent exposure of negative control and indirect contact reindeer to potential infectivity on feed and water buckets, bedding, or fencing $(6,11)$. However, reindeer 


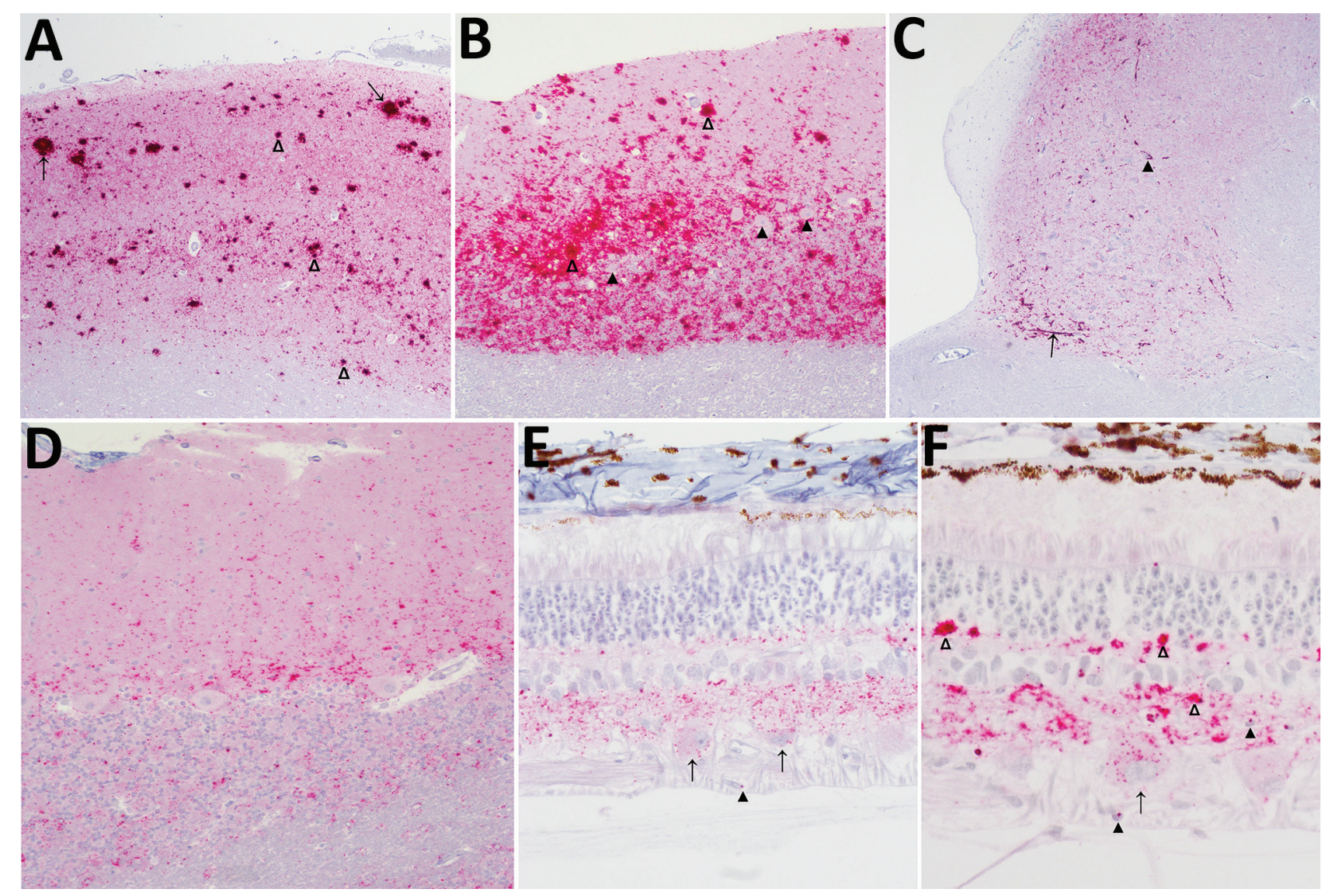

Figure 1. Immunohistochemical analysis for the prion protein showing scrapie prion protein $(P r P s c)$ deposits in brains $(A-D)$ and retinas $(\mathrm{E}, \mathrm{F})$ from reindeer (Rangifer tarandus tarandus) with chronic wasting disease. PrPsc immunodetection using the monoclonal antibody F99/97.6.1. A) Neocortex, showing prominent aggregated (open arrowheads) and plaque-like (arrows) deposits in reindeer no. 4. Original magnification $\times 5$. B) Cerebellum, showing particulate immunoreactivity and aggregated deposits (open arrowheads) in reindeer no. 4. Note absence of intraneuronal immunoreactivity in Purkinje cells (solid arrowheads). Original magnification $\times 10$. C) Brainstem at the level of the obex, showing prominent linear (arrow) and perineuronal (solid arrowhead) immunoreactivity in the dorsal motor nucleus of the vagus nerve in reindeer no. 21. Original magnification $\times 5$. D) Cerebellum, punctate immunoreactivity in the molecular and granular layers and white matter in reindeer no. 12. Original magnification $\times 5$. E) Intraneuronal immunoreactivity in retinal ganglion cells (arrows), punctate deposits in the inner and outer plexiform layers, scattered intramicroglial deposits (solid arrowheads) in reindeer no. 12 . Original magnification $\times 40$. F) Particulate to coalescing deposits in the inner and outer plexiform layers (open arrowheads), intraneuronal immunoreactivity in retinal ganglion cells (arrows), and scattered intramicroglial deposits (solid arrowheads) in reindeer no. 13. Original magnification $\times 40$.

might have had access to bedding from adjacent pens that had spread into the central alleyway.

During the 5-year course of this study, reindeer were moved between pens several times to maintain an optimal number of animals per pen (online Technical Appendix Figure 1). Prolonged persistence of prion infectivity in the natural environment has been documented for both CWD (2 years [5]) and scrapie (up to 16 years [12]). In addition, thorough cleaning and disinfection might not be sufficient to remove all infectivity from the environment, leading to persistence of infectivity under experimental housing conditions (13).

In reindeer challenged orally with the agent of CWD, the SS138 genotype (serine/serine at PRNP codon 138) has been associated with susceptibility to disease and the
NS138 (asparagine/serine) genotype with resistance (1). In the study we report, disease developed in reindeer with the NS138 genotype after intracranial inoculation, although the extent of lymphoreticular system involvement was significantly lower than in NN138 and SS138 reindeer. The potential association of the NN138 polymorphism with shorter survival times is interesting. However, as with all potential genotype versus phenotype interactions, care should be taken not to over-interpret these results given the small group sizes and the large number of $P R N P$ genotype groups in this study.

Our results demonstrate that reindeer are susceptible to the agent of CWD from white-tailed deer, mule deer, and elk sources after intracranial inoculation. Furthermore, naive reindeer are susceptible to the agent of CWD after 
Figure 2. Western blot characterization of the inocula used to inoculate reindeer and brainstem samples from representative reindeer from each experimental group in study of chronic wasting disease transmission. Scrapie prion protein $\left(\mathrm{PrP}^{\mathrm{Sc}}\right)$ immunodetection using the monoclonal antibody 6H4. Positive Western blot results demonstrate a 3-band pattern (diglycosylated, highest; monoglycosylated, middle; and nonglycosylated, lowest) that is characteristic of prion diseases. Lanes: 1, biotinylated protein marker; 2 and 3, indirect contact reindeer (animals no. 20 and 21, respectively); 4 and 5, reindeer inoculated intracranially with CWD ${ }^{\mathrm{md}}$ (animals no. 15 and 12 respectively); 6, CWD ${ }^{\mathrm{md}}$ inoculum; 7, direct contact reindeer (no. 7, cohoused with CWD wid-inoculated reindeer); 8, reindeer (no. 5) inoculated intracranially with CWD ${ }^{\text {wtd }}$; 9 , CWD ${ }^{\text {wtd }}$ inoculum; 10, reindeer (no. 10) inoculated intracranially with CWD elk; 11, CWD elk inoculum; 12, marker. CWD, chronic wasting disease; CWD ${ }^{\text {ek }}$, CWD-affected elk; CWD ${ }^{\text {md }}$, CWD-affected mule deer; CWD ${ }^{\text {wtd }}$, CWD-affected white-tailed deer combined with brain material from experimentally challenged white-tailed deer.

direct and indirect exposure to CWD-infected reindeer, suggesting a high potential for horizontal transmission of CWD within and between farmed and free-ranging reindeer (and caribou) populations.

\section{Acknowledgments}

We thank Martha Church, Robyn Kokemuller, Joe Lesan, Virginia Montgomery, Dennis Orcutt, and Trudy Tatum for excellent technical support.

Dr. Moore is a postdoctoral research associate at the National Animal Disease Center, US Department of Agriculture, Ames, Iowa. Her research interests include pathogenesis and pathology of animal diseases with a special interest in neuropathology and prion diseases.

\section{References}

1. Mitchell GB, Sigurdson CJ, O'Rourke KI, Algire J, Harrington NP, Walther I, et al. Experimental oral transmission of chronic wasting disease to reindeer (Rangifer tarandus tarandus). PLoS One. 2012;7:e39055. http://dx.doi.org/10.1371/journal. pone.0039055

2. Norwegian Veterinary Institute. The first detection of chronic wasting disease (CWD) in Europe. 2016 April 4 [cited 2016 Apr 5]. http://www.vetinst.no/sykdom-og-agens/chronic-wasting-disease/ the-first-detection-of-chronic-wasting-disease-cwd-in-europe

3. Becker R. Deadly animal prion disease appears in Europe. 2016 [cited 2016 Jun 16]. http://www.nature.com/news/deadly-animalprion-disease-appears-in-europe-1.19759

4. Haley NJ, Hoover EA. Chronic wasting disease of cervids: current knowledge and future perspectives. Annu Rev Anim Biosci. 2015;3:305-25. http://dx.doi.org/10.1146/annurevanimal-022114-111001
5. Miller MW, Williams ES, Hobbs NT, Wolfe LL. Environmental sources of prion transmission in mule deer. Emerg Infect Dis. 2004;10:1003-6. http://dx.doi.org/10.3201/eid1006.040010

6. Henderson DM, Denkers ND, Hoover CE, Garbino N, Mathiason CK, Hoover EA. Longitudinal detection of prion shedding in saliva and urine by CWD-infected deer by real-time quaking-induced conversion. J Virol. 2015;89:9338-47. http://dx.doi.org/10.1128/JVI.01118-15

7. Greenlee JJ, Smith JD, Kunkle RA. White-tailed deer are susceptible to the agent of sheep scrapie by intracerebral inoculation. Vet Res. 2011;42:107. http://dx.doi.org/10.1186/1297-9716-42-107

8. Mathiason CK, Hays SA, Powers J, Hayes-Klug J, Langenberg J, Dahmes SJ, et al. Infectious prions in pre-clinical deer and transmission of chronic wasting disease solely by environmental exposure. PLoS One. 2009;4:e5916. http://dx.doi.org/10.1371/ journal.pone. 0005916

9. Tamgüney G, Richt JA, Hamir AN, Greenlee JJ, Miller MW, Wolfe LL, et al. Salivary prions in sheep and deer. Prion. 2012;6:52-61. http://dx.doi.org/10.4161/pri.6.1.16984

10. Gough KC, Baker CA, Simmons HA, Hawkins SA, Maddison BC. Circulation of prions within dust on a scrapie affected farm. Vet Res. 2015;46:40. http://dx.doi.org/10.1186/s13567-015-0176-1

11. Maddison BC, Baker CA, Terry LA, Bellworthy SJ, Thorne L, Rees HC, et al. Environmental sources of scrapie prions. J Virol. 2010;84:11560-2. http://dx.doi.org/10.1128/JVI.01133-10

12. Georgsson G, Sigurdarson S, Brown P. Infectious agent of sheep scrapie may persist in the environment for at least 16 years. J Gen Virol. 2006;87:3737-40. http://dx.doi.org/10.1099/vir.0.82011-0

13. Hawkins SA, Simmons HA, Gough KC, Maddison BC. Persistence of ovine scrapie infectivity in a farm environment following cleaning and decontamination. Vet Rec. 2015;176:99. http://dx.doi.org/10.1136/vr.102743

Address for correspondence: Justin Greenlee, Virus and Prion Disease Research Unit, National Animal Disease Center, Agricultural Research Service, US Department of Agriculture, 1920 Dayton Ave, Ames, IA 50010, USA; email: justin.greenlee@ars.usda.gov 